\title{
Can we trust in Sars-CoV-2 rapid antigen testing? Preliminary results from a paediatric cohort in the emergency department
}

\author{
Marco Denina $^{1,2}$ (D) $\cdot$ Virginia Giannone ${ }^{3} \cdot$ Antonio Curtoni $^{4} \cdot$ Elisa Zanotto $^{4} \cdot$ Silvia Garazzino $^{2}$. \\ Antonio Francesco Urbino ${ }^{1}$. Claudia Bondone ${ }^{1}$
}

Received: 2 September 2021 / Accepted: 7 September 2021 / Published online: 14 September 2021

(c) Royal Academy of Medicine in Ireland 2021

\begin{abstract}
Background Rapid identification of Covid-19 in the paediatric emergency department is critical; Antigen tests are fast but poorly investigated in children.

Aims To investigate Sars-CoV-2 antigen rapid test in children.

Methods We compare the performance of LumiraDx with molecular tests in a paediatric emergency department.

Results A retrospective cohort of 191 patients with AT and PCR tests performed in the same episode was analysed; $16 \%$ resulted positive for Sars-CoV-2. Using the PCR test as the gold standard, we calculated antigen testing overall sensitivity of $94.1 \%$, specificity of $91.9 \%$, and NPV of $99.4 \%$. Only one false-negative test was found.

Conclusions AT may be helpful in the initial screening of patients at PED.
\end{abstract}

Keywords Antigen tests · COVID-19 · Emergency department · Point-of-care tests · Sars-Cov-2

The rapid and precise identification of COVID-19 patients in the emergency department (ED) is critical for both correctly diagnosing COVID-19 patients and preventing the virus diffusion into the hospital. Furthermore, the variable clinical presentation of COVID-19 in paediatric age is well described, with signs and symptoms that overlap most viral infections, making SARS-CoV-2 detection crucial in the paediatric ED (PED) [1]. The principal limit of the RealTime Polymerase Chain Reaction test (PCR) is that results are often delayed - some hours from the swab collecting to the final report - which is not appropriate for the ED timing. Antigen testing (AT) is rapid (about $15 \mathrm{~min}$ ) and may represent an optimal resource in an emergency setting [2].
However, data about sensitivity and specificity in paediatric age are scarce.

We retrospectively analysed all PCR and AT performed in the PED of the Regina Margherita Children hospital, in Turin, from October 23, 2020 to January 23, 2021. We selected only the patients tested with AT and PCR in the same episode, collecting clinical and epidemiological data. All patients with risk factors for SARS-CoV-2 infection (clinical symptoms and/or epidemiological link with COVID-19 patient) and all patients who required hospitalisation were tested by PCR. AT test was performed in all patients, regardless of symptoms or epidemiological link, who needed urgent access to diagnostic service outside the

Claudia Bondone

claudiabondone@virgilio.it

1 Department of Pediatric Emergency, Regina Margherita Children's Hospital, Turin, Italy

2 Department of Pediatrics, Infectious Diseases Unit, University of Turin, Regina Margherita Children's Hospital, Turin, Italy

3 Department of Pediatrics and Public Health, University of Turin, Regina Margherita Children's Hospital, Turin, Italy

4 Department of Public Health and Pediatrics, Microbiology and Virology Unit, Molinette Hospital, University of Turin, Città della salute e della Scienza, Turin, Italy 
PED before the PCR result was available. No institutional review board approval was needed for this study.

Data were statistically analysed using SPSS Statistic v23 (IBM, Armonk, NY). Continuous variables with non-normal distribution were expressed as medians (interquartile ranges), and categorical variables were expressed as numbers (percentages) and were compared with the $\chi^{2}$ test or Fisher exact test. A two-sided $p$ value $<0.05$ was considered statistically significant. Agreement between techniques was calculated using Cohen kappa coefficient $(\kappa)$ [3].

Antigen tests were performed with LumiraDx ${ }^{\mathrm{TM}}$ Platform (LumiraDx UK Ltd., Alloa, UK), a microfluidic immunofluorescence-based assay with a reported specificity of $96.6 \%$ and sensitivity of $97.6 \%$ [4]. The LumiraDx Sars-CoV-2 Antigen Test uses SARS-CoV/Sars-CoV-2 specific antibodies in a particle-particle sandwich immunoassay to determine the presence of Sars-CoV-2 Nucleocapsid Protein antigen present in the test sample. Molecular tests were performed with Simplexa ${ }^{\mathrm{TM}}$ COVID19 Direct kit (Diasorin, Saluggia, Italy); the PCR assay targets two different regions SARS-CoV-2 genome, the ORF1 ab and S (spike) gene. DiaSorin Molecular conducted an in-silico evaluation of Simplexa ${ }^{\mathrm{TM}}$ COVID-19 Direct kit towards the UK isolated SARS-CoV-2 virus variant (variant B.1.1.7), confirming that the analytical performance of the assay remains unchanged. According to manufacturer instructions, PCR tests were performed on a flocked nasopharyngeal swab and AT on a flocked nasal swab, collected from both nostrils in the same moment by the same operator.

As for the majority of Italian hospitals, our PED was organised in two areas ("COVID-19 free" and "COVID-19 possible") based on a pre-triage survey of patients and caregivers. Based on the totality of PCR tests performed during the study period, the prevalence of COVID-19 was 5\% in the "COVID-19-free department" and 12\% in the "COVID19-possible department".

We collected 191 patients with both diagnostic tests performed in the same PED access in the study period. Of them, 31 had at least one positive test (PCR or AT). No symptoms were related to a positive SARS-CoV-2 AT, as shown in Table 1.

The majority of samples were collected during the initial phase of the disease, with a median onset of symptoms of 1 day (IQR: $1-4)$. Eighty children (42\%) were admitted, and 111 (58\%) were discharged from PED; SARS-CoV-2 infection was not related to hospitalisation $(p=0.24)$.

Assuming PCR test as the gold standard, amongst 191 cases, we identified 1 false-negative and 14 false-positive ATs. PCR in discordant couples of tests was repeated within $48 \mathrm{~h}$, and in all 15 cases confirmed the previous PCR test results.

We obtained for AT an overall sensitivity of $94.1 \%$ (95\% CI: 71.3 to $99.8 \%$ ) and specificity of $91.9 \%$ (95\%
CI: $86.9 \%$ to $95.5 \%$ ) with a PPV of $52.1 \%$ and an NPV of $99.4 \%$. Results showed a substantial agreement with a $\kappa$ for the LumiraDx test of 0.64 [3].

These results allow some reflections. Diagnostic test interpretation in PED must include clinical and epidemiological data [5]. PCR tests are considered the gold standard for diagnosing SARS-CoV-2 but are subject to false results mostly related to specimen collection timing or errors and the influence of the disease's prevalence in the population. Interestingly, despite the study taking place during the second wave of COVID-19, the prevalence in our PED has remained consistently low. Considering the recent progress in vaccination campaigns and the arrival of the warm season, we expect a similar, or even better, context during the next months in PEDs.

In light of this, our preliminary results may be useful: on the one hand, the high sensitivity and NPV seem to suggest that we can trust in a negative AT. However, on the other hand, despite a high specificity, a positive AT must be confirmed with a PCR test due to the low PPV when performed in a low prevalence setting as a PED.

One issue to discuss is the presence, in our cohort, of 14 positive ATs that tested negative at PCR. In symptomatic patients, despite the suppressed circulation of other viruses by social distancing and prevention measures, we may hypothesise in some cases a cross-reaction of the test with other respiratory viruses. Unfortunately, these patients were not tested for other pathogens (such as other coronaviruses or influenza). On the other side, in asymptomatic patients screened in the COVID-19-free department, false positives AT may be related to the disease's low prevalence.

Furthermore, epidemiological context must be considered: in Italy, in the last part of 2020, the circulation of several variants of the SARS-CoV-2 virus was documented. Both the so-called UK variant and the Brazilian variant have several mutations in the spike protein, but this should not cause antigen tests problems, as these detect the $\mathrm{N}$ protein. Moreover, new different mutations are emerging for the $\mathrm{N}$ protein, and this should be carefully monitored to assess the possible influence on antigen tests that use it as a target.

Our test is microfluidic immunofluorescence-based and is currently classified as a last-generation test, theoretically with characteristics comparable with PCR.

Instead we found in the literature that only two studies focus on rapid AT in PED, both using a first-generation antigen test, working with a lateral flow method (PanbioABBOTT) $[6,7]$.

Both studies highlight AT's excellent specificity in front of low sensitivity, making it necessary to check every negative AT in patients with some COVID-19-associated symptoms that in paediatric age represent the majority of patients in PED. 
Table 1 Epidemiological and clinical features of paediatric patients in PED, followed by the contingency tables in which the performance of the AT is compared with PCR

\begin{tabular}{|c|c|c|c|}
\hline & Sars-Cov-2 positive AT & Sars-Cov-2 negative AT & $p$ \\
\hline Female patients, $n(\%)$ & $19(63.3 \%)$ & $69(42.8 \%)$ & 0.077 \\
\hline Male patients, $n(\%)$ & $11(36.7 \%)$ & $92(57.2 \%)$ & \\
\hline Median age, years (IQR) & $5.8(1.1-10.8)$ & $4.5(1.4-9.8)$ & 0.74 \\
\hline \multicolumn{4}{|l|}{ Outcome } \\
\hline Admitted, $n(\%)$ & $15(50.0 \%)$ & $65(40.3 \%)$ & 0.41 \\
\hline Discharged from emergency department, $n(\%)$ & $15(50.0 \%)$ & $96(59.7 \%)$ & \\
\hline Symptoms, $n(\%)$ & $21(71.0 \%)$ & $134(83.2 \%)$ & 0.12 \\
\hline Fever (body temperature $\left.>37.5^{\circ} \mathrm{C}\right), n(\%)$ & $11(36.6 \%)$ & $75(46.6 \%)$ & 0.42 \\
\hline Dry cough, $n(\%)$ & $6(20.0 \%)$ & $29(18.0 \%)$ & 0.80 \\
\hline Diarrhoea, $n(\%)$ & $2(6.7 \%)$ & $25(15.5 \%)$ & 0.26 \\
\hline Dyspnoea, $n(\%)$ & 0 & $17(10.5 \%)$ & 0.08 \\
\hline Sore throat, $n(\%)$ & $1(3.3 \%)$ & $4(2.5 \%)$ & 0.58 \\
\hline Smell and taste dysfunction, $n(\%)$ & 0 & 0 & \\
\hline Abdominal pain, $n(\%)$ & $3(10 \%)$ & $42(26.1 \%)$ & 0.06 \\
\hline Vomiting, $n(\%)$ & $7(23.3 \%)$ & $53(32.9 \%)$ & 0.39 \\
\hline Rhinitis, $n(\%)$ & $3(10 \%)$ & $29(18.0 \%)$ & 0.30 \\
\hline Hyporexia, $n(\%)$ & $3(10 \%)$ & $23(14.3 \%)$ & 0.77 \\
\hline Conjunctivitis, $n(\%)$ & 0 & $1(0.6 \%)$ & 1 \\
\hline Neurological manifestations, $n(\%)$ & $2(6.7 \%)$ & $19(11.8 \%)$ & 0.54 \\
\hline Headache, $n(\%)$ & $2(6.7 \%)$ & $8(5.0 \%)$ & 0.66 \\
\hline \multicolumn{4}{|l|}{$2 \times 2$ contingency table including all patients } \\
\hline & & PCR positive & PCR negative \\
\hline & AT positive, $n$ & 16 & 14 \\
\hline & AT negative, $n$ & 1 & 160 \\
\hline Sensitivity & $94.1 \%$ & & \\
\hline Specificity & $91.9 \%$ & & \\
\hline Positive likelihood ratio & 11.7 & & \\
\hline Negative likelihood ratio & 0.06 & & \\
\hline PPV & $52.0 \%$ & & \\
\hline NPV & $99.4 \%$ & & \\
\hline Accuracy & $92.1 \%$ & & \\
\hline $\mathrm{K}$ & 0.63 & & \\
\hline
\end{tabular}

Unlike those two reports, our data suggested that a negative rapid AT performed in a SARS-CoV-2 low-prevalence setting, as a PED, has a very low probability of being a false negative test, simplifying diagnostic and care pathways in PED.

The present study has some limitations. First, the number of asymptomatic children is small, and the prevalence of COVID-19 in this category is very low, limiting the analysis in this group of patients. Secondly, the absence of data on alternative infections/coinfections in false-positive patients makes these data difficult to interpret.

Despite this, our preliminary data suggest that in the PED's fast-paced setting, the use of rapid antigen test as a first point-of-care screening, later integrated with molecular test, may be helpful, time-saving and cost-reducing, allowing a simplification of diagnostic and care pathways. A direct consequence may be reducing the time children stay in the waiting room and into the PED, reducing the possibility of in-hospital infection with SARS-CoV-2 or other pathogens.

In conclusion, if future studies confirm the present preliminary results, this could lead to a change in rapid AT use in PED as an initial screening to simplify and rapidly refer patients to diagnostic or care pathways whilst awaiting molecular tests.

Author contribution $\mathrm{MD}$ and $\mathrm{CB}$ gave substantial contribution to conception and design, drafted the article, reviewed and critically revised the manuscript; MD made statistical analysis; VG, AC, EZ, SG and 
AFU contributed to conception and design, collected data and revised the manuscript; all authors approved the final version of the manuscript and agree to be accountable for all aspects of the work.

Availability of data and material Not applicable.

Code availability Not applicable.

\section{Declarations}

Conflict of interest The authors declare no competing interests.

\section{References}

1. Denina M, Aguzzi S, Versace A et al (2021) Testing strategy for SARS-CoV-2 in the paediatric emergency department. Arch Dis Child 106:e11. https://doi.org/10.1136/archdischild-2020-319806

2. Turcato G, Zaboli A, Pfeifer $\mathrm{N}$ et al (2021) Clinical application of a rapid antigen test for the detection of SARS-CoV-2 infection in symptomatic and asymptomatic patients evaluated in the emergency department: a preliminary report. J Infect 82:e14-e16. https://doi.org/10.1016/j.jinf.2020.12.012
3. Landis JR, Koch GG (1977) The measurement of observer agreement for categorical data. Biometrics 33:159-174. https://doi.org/ $10.2307 / 2529310$

4. Kohmer N, Toptan T, Pallas C et al (2021) The comparative clinical performance of four SARS-CoV-2 rapid antigen tests and their correlation to infectivity in vitro. J Clin Med 10.https://doi.org/10. 3390/jcm 10020328

5. Pivetta E, Goffi A, Tizzani M et al (2021) Lung ultrasonography for the diagnosis of SARS-CoV-2 pneumonia in the emergency department. Ann Emerg Med 77:385-394. https://doi.org/10. 1016/j.annemergmed.2020.10.008

6. González-Donapetry P, García-Clemente P, Bloise I et al (2021) Think of the children: evaluation of SARS-CoV-2 rapid antigen test in pediatric population. Pediatr Infect Dis J. https://doi.org/ 10.1097/INF.0000000000003101

7. Villaverde S, Domínguez-Rodríguez S, Sabrido G et al (2021) Diagnostic accuracy of the Panbio severe acute respiratory syndrome coronavirus 2 antigen rapid test compared with reversetranscriptase polymerase chain reaction testing of nasopharyngeal samples in the pediatric population. J Pediatr. https://doi.org/10. $1016 /$ j.jpeds.2021.01.027

Publisher's Note Springer Nature remains neutral with regard to jurisdictional claims in published maps and institutional affiliations. 